\title{
GENERALIZATION OF CERTAIN SECOND ORDER ORDINARY DIFFERENTIAL EQUATION OF NISHIMOTO BY THE FRACTIONAL CALCULUS METHOD
}

\section{SHIH-TONG TU AND KATSUYUKI NISHIMOTO}

\begin{abstract}
A generalization of theorems derived by Nishimoto ([4], [8] ${ }^{a}$ pp. 154-161), for certain second order ordinary differential equation of Fuchs type was reported by S. Owa. K. Nishimoto and their colleagues [7]. In this paper, a new proof for generalization theorems in previous paper and the second generalization theorems for the second order ordinary differential equation of Nishimoto are reported.
\end{abstract}

$\S 0$. Introduction (Definition of fractional calculus)

\section{Definition.}

Let $D=\{\underline{D}, \underline{D}\}, C=\{\underline{C}, \underset{+}{C}\}$,

$C$ be a curve along the cut joining two points $z$ and $-\infty+i \operatorname{Im}(z)$,

$\bar{C}$ be a curve along the cut joining two points $z$ and $\infty+i \operatorname{Im}(z)$,

$\underline{D}$ be a domain surrounded by $\underline{C}$,

$\underset{t}{D}$ be a domain surrounded by $\underset{+}{C}$.

(Here $D$ contains the points over the curves $C^{\prime}$ )

Received October 19, 1990.

1980 Mathematics Subject Classification (1985 Revision).

Primary 26A30; Secondary 34A30.

This work was supported, in part, by the National Science Council of R.O.C. under Grant NSC 80-0208M033-01. 
Moreover, let $f=f(z)$ be a regular function in $D(z \in D)$,

$$
\begin{gathered}
f_{\nu}=(f)_{\nu}={ }_{c}(f)_{\nu}=\frac{\Gamma(\nu+1)}{2 \pi i} \int_{c} \frac{f(\zeta)}{(\zeta-z)^{\nu+1}} d \zeta \quad\left(\nu \notin \mathbb{Z}^{-}\right) \\
(f)_{-m}=\lim _{\nu \rightarrow-m}(f)_{\nu} \quad\left(m \in \mathbb{Z}^{+}\right),
\end{gathered}
$$

where $-\pi \leq \arg (\zeta-z) \leq \pi$ for $\underline{C}, 0 \leq \arg (\zeta-z) \leq 2 \pi$ for $\underset{+}{C}, \zeta \neq z, \Gamma$; Gamma function.

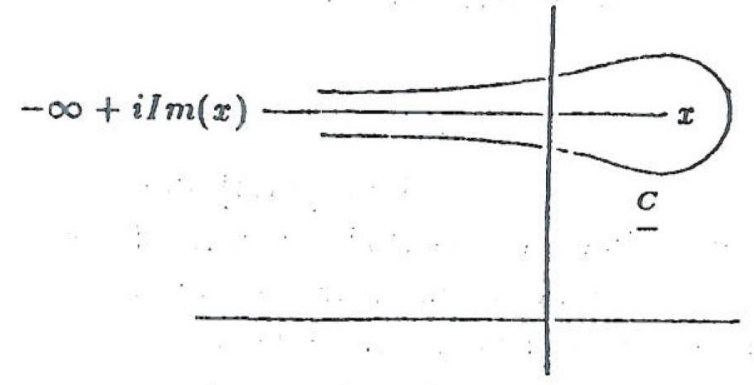

Fig. 1.

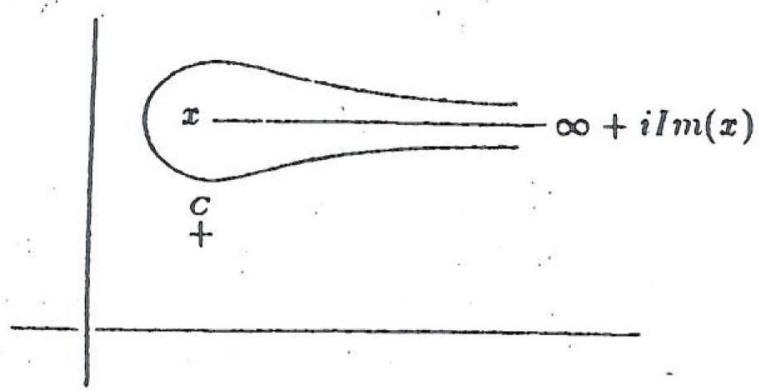

Fig. 2.

then $(f)_{\nu}$ is the fractional differintegration of arbitrary order $\nu$ (derivatives of order $\nu$ for $\nu>0$, and integrals of order $-\nu$ for $\nu<0$ ), with respect to $z$, of the function $f$ if $\left|(f)_{\nu}\right|<\infty$.

Note 1. Consider the principal value for many valued function $f$

Note 2. For the complex $\nu$, we consider the principal value for our convenience.

Note 3.

$$
f_{\nu}=(f)_{\nu} \text { is } \begin{cases}\text { derivative } & \text { for } \operatorname{Re}(\nu)>0 \\ \text { original } & \text { for } \nu=0 \\ \text { integral } & \text { for } \operatorname{Re}(\nu)<0\end{cases}
$$

for $\nu \in C$, if $f_{\nu}$ exists.

And in case of $\operatorname{Re}(\nu)=0, f_{\nu}$ is only formal differintegration regardless of $\operatorname{Im}(\nu) \geq 0$. That is, we have no derivative and integral for $\nu=$ pure imaginary.

§1. Theorems for a second order ordinary differential equation of Nishimoto Nishimoto gave the following results by the method of fractional calculus [4]. 
Theorem A. If $f_{\alpha}(\neq 0)$ exists, then the differential equation of Fuchs type

$$
\varphi_{2} \cdot z^{2}+\varphi_{1} \cdot 2 \alpha z+\varphi \cdot \alpha(\alpha-1)=f \quad(z \neq 0)
$$

has a particular solution of the form

$$
\varphi=\left(f_{-\alpha} \cdot z^{-2}\right)_{\alpha-2}
$$

where $\varphi=\varphi(z), \alpha(\in \mathbb{C})$ is a given constant, $z \in \mathbb{C}$ and $f=f(z)$ is known.

Theorem B. The differential equation of Fuchs type

$$
\varphi_{2} \cdot z^{2}+\varphi_{1} \cdot 2 \alpha z+\varphi \cdot \alpha(\alpha-1)=0 \quad(z \neq 0)
$$

has a solution

$$
\varphi=K\left(z^{-2}\right)_{\alpha-2}=K e^{-i \pi \alpha} \Gamma(\alpha) z^{-\alpha}
$$

where $\alpha \notin \mathbb{Z}^{-} \cup\{0\}, K$ is an arbitrary constant of the integration, $\varphi=\varphi(z)$ and $z \in \mathrm{C}$.

Theorem $\mathrm{C}$. If $f_{\alpha}(\neq 0)$ exists, then the differential equation given by (1.1) has the solution

$$
\begin{aligned}
\varphi & =\left(f_{-\alpha} \cdot z^{-2}\right)_{\alpha-2}+K\left(z^{-2}\right)_{\alpha-2} \\
& =\left(f_{-\alpha} \cdot z^{-2}\right)_{\alpha-2}+K e^{-i \pi \alpha} \Gamma(\alpha) z^{-\alpha}
\end{aligned}
$$

where $\alpha \notin \mathbb{Z}^{-} \cup\{0\}, K$ is an arbitrary constant of the integration, $\varphi=\varphi(z)$, $f=f(z)$ is known, and $z \in \mathbf{C}$.

§2. A Generalization of Nishimoto's results in $\S 1$

The following theorems were derived by $S$. Owa, K. Nishimoto and their colleague [7] by means of fractional calculus. The same theorems are treated here again. "However the method to obtain these theorems, which is described as below, is different from the former slightly. 
Theorem 1. If $f_{\alpha}(\neq 0)$ exists, then the nonhomogeneous differential equation of Fuchs type

$$
\begin{aligned}
L[\varphi(z), n, \alpha] \equiv \varphi_{n} \cdot & z^{n}+\sum_{k=1}^{n} \varphi_{n-k} \cdot\left\{\frac{\Gamma(\alpha)}{\Gamma(\alpha-k) \Gamma(k+1)}\left(z^{n}\right)_{k}\right. \\
& \left.+\frac{\Gamma(\alpha) n}{\Gamma(\alpha+1-k) \Gamma(k)}\left(z^{n-1}\right)_{k-1}\right\}=f \quad(z \neq 0)
\end{aligned}
$$

has a particular solution of the form

$$
\varphi=\left(f_{-\alpha} \cdot z^{-n}\right)_{\alpha-n}
$$

where $\varphi=\varphi(z), n \in \mathbb{Z}^{+}, \alpha(\in \mathbb{C})$ is a given constant, $z \in \mathbf{C}$ and $f=f(z) \neq 0$ is known.

Proof. Let

$$
\varphi=w_{\alpha} \quad\left(\varphi_{0}=\varphi\right)
$$

hence

$$
\varphi_{n}=w_{\alpha+n}
$$

we have then

$$
\begin{gathered}
w_{\alpha+n} \cdot z^{n}+\sum_{k=1}^{n} w_{\alpha+n-k}\left\{\frac{\Gamma(\alpha)}{\Gamma(\alpha-k) \Gamma(k+1)}\left(z^{n}\right)_{k}\right. \\
\left.+\frac{\Gamma(\alpha) n}{\Gamma(\alpha+1-k) \Gamma(k)}\left(z^{n-1}\right)_{k-1}\right\}=f
\end{gathered}
$$

from (2.1). Since

$$
\begin{aligned}
\left(w_{n+1} \cdot z^{n}\right)_{\alpha-1} & =\sum_{k=0}^{n} \frac{\Gamma(\alpha)}{\Gamma(\alpha-k) \Gamma(k+1)}\left(w_{n+1}\right)_{\alpha-1-k}\left(z^{n}\right)_{k} \\
& =w_{n+\alpha} \cdot z^{n}+\sum_{k=1}^{n} \frac{\Gamma(\alpha)}{\Gamma(\alpha-k) \Gamma(k+1)} w_{n+\alpha-k}\left(z^{n}\right)_{k}
\end{aligned}
$$

and 


$$
\begin{aligned}
\left(w_{n} \cdot z^{n-1}\right)_{\alpha-1} & =\sum_{k=0}^{n-1} \frac{\Gamma(\alpha)}{\Gamma(\alpha-k) \Gamma(k+1)}\left(w_{n}\right)_{\alpha-1-k}\left(z^{n-1}\right)_{k} \\
& =\sum_{k=1}^{n} \frac{\Gamma(\alpha)}{\Gamma(\alpha+1-k) \Gamma(k)} w_{n+\alpha-k}\left(z^{n-1}\right)_{k-1}
\end{aligned}
$$

(2.4) gives

$$
\left(w_{n+1} \cdot z^{n}\right)_{\alpha-1}+n\left(w_{n} \cdot z^{n-1}\right)_{\alpha-1}=f
$$

that is,

$$
w_{n+1} \cdot z^{n}+n w_{n} \cdot z^{n-1}=f_{1-\alpha}
$$

This is equivalent to

$$
\left(w_{n} \cdot z^{n}\right)_{1}=f_{1-\alpha}
$$

Thus we have

$$
w=\left(f_{-\alpha} \cdot z^{-n}\right)_{-n}
$$

or

$$
\varphi=w_{\alpha}=\left(f_{-\alpha} \cdot z^{-n}\right)_{\alpha-n}
$$

as a particular solution to the differential equation (2.1).

Conversely, we have

$$
\varphi_{n}=\left(f_{-\alpha} \cdot z^{-n}\right)_{\alpha}=w_{\alpha+n}
$$

then substituting this into the left hand side of (2.1), we obtain

$$
\begin{aligned}
& \text { L. H. S. of }(2.1)=w_{\alpha+n} \cdot z^{n} \\
& \quad+\sum_{k=1}^{n} w_{\alpha+n-k} \cdot\left\{\frac{\Gamma(\alpha)}{\Gamma(\alpha-k) \Gamma(k+1)}\left(z^{n}\right)_{k}+\frac{\Gamma(\alpha) n}{\Gamma(\alpha+1-k) \Gamma(k)}\left(z^{n-1}\right)_{k-1}\right\} \\
& =\left(w_{n+1} \cdot z^{n}\right)_{\alpha-1}+n\left(w_{n} \cdot z^{n-1}\right)_{\alpha-1} \\
& =\left(w_{n+1} \cdot z^{n}+n w_{n} \cdot z^{n-1}\right)_{\alpha-1} \\
& =\left(\left(f_{-\alpha} \cdot z^{-n}\right)_{1} \cdot z^{n}+n \cdot f_{-\alpha} \cdot z^{-n} \cdot z^{n-1}\right)_{\alpha-1} \\
& =\left(f_{1-\alpha} \cdot z^{-n} \cdot z^{n}+f_{-\alpha} \cdot\left(z^{-n}\right)_{1} \cdot z^{n}+n \cdot f_{-\alpha} \cdot z^{-1}\right)_{\alpha-1} \\
& =\left(f_{1-\alpha}\right)_{\alpha-1} \\
& =f .
\end{aligned}
$$


Note 1. Letting $n=2$ in Theorem 1, we have Theorem A in $\S 1$.

Note 2. The treatment described above is better than previous one.

Theorem 2. Homogeneous differential equation of Fuchs type

$$
L[\varphi(z), n, \alpha]=0 \quad(z \neq 0)
$$

has a solution

$$
\varphi=K\left(z^{-n}\right)_{\alpha-n}=K e^{-i \pi(\alpha-n)} \frac{\Gamma(\alpha)}{\Gamma(n)} \cdot z^{-\alpha},
$$

where $n \in Z^{+}, \alpha \notin \mathbb{Z}^{-} \cup\{0\}, z \in \mathbb{C}, \varphi=\varphi(z)$, and $K(\neq 0)$ is an arbitrary constant.

Proof. In the same way with the proof of previous theorem, with the aid of (2.3), (2.5) and (2.6) we obtain

$$
w_{n+1} \cdot z^{n}+n w_{n} \cdot z^{n-1}=0
$$

that is,

$$
\frac{w_{n+1}}{w_{n}}=-n z^{-1}
$$

from (2.13). Integrating bothe sides in (2.15),we have

$$
w_{n}=K z^{-n}, \quad(K \neq 0)
$$

hence

$$
\begin{aligned}
\varphi=w_{\alpha} & =K\left(z^{-n}\right)_{\alpha-n} \\
& =K e^{-i \pi(\alpha-n)} \frac{\Gamma(\alpha)}{\Gamma(n)} z^{-\alpha},
\end{aligned}
$$

as a solution to the homogeneous equation (2.13), where $\alpha \notin \mathbb{Z}^{-} \cup\{0\}$.

Conversely, substituting (2.16) into the left hand side of (2.13), we get

$$
\begin{aligned}
\text { L. II. S. of (2.13) } & =\left(w_{n+1} \cdot z^{n}\right)_{\alpha-1}+n\left(w_{n} \cdot z^{n-1}\right)_{\alpha-1} \\
& =\left(w_{n+1} \cdot z^{n}+n w_{n} \cdot z^{n-1}\right)_{\alpha-1} \\
& =\left(\left(K z^{-n}\right)_{1} \cdot z^{n}+n K z^{-n} \cdot z^{n-1}\right)_{\alpha-1} \\
& =(0)_{\alpha-1} \\
& =0 .
\end{aligned}
$$


Note. Taking $n=2$, theorem 2 derives theorem $\mathrm{B}$ in $\S 1$.

Theorem 3. If $f_{\alpha}(\neq 0)$ exists, then the differintegrated function

$$
\varphi=\left(f_{-\alpha} \cdot z^{-n}\right)_{\alpha-n}+K\left(z^{-n}\right)_{\alpha-n}
$$

satisfies the differential equation of Fuchs type (2.1), where $n \in \mathbb{Z}^{+}, \alpha \notin \mathbb{Z}^{-} \cup\{0\}$, $z \in \mathbb{C}, \varphi=\varphi(z), f=f(z)$ is known, and $K(\neq 0)$ is an arbitrary constant.

Proof. It is clear by the theorems 1 and 2 .

Note. Taking $n=2$ in theorem 3 , we have theorem $\mathrm{C}$ in $\S 1$.

$\S 3$. The second generalization of Nishimoto's results in $§ 1$.

THeorem 4. If $f_{\alpha}(\neq 0)$ exists, then the nonhomogeneous differintegral equation of Fuchs type

$$
\begin{aligned}
L[\varphi(z), m, n, \alpha] \equiv & \varphi_{m} \cdot z^{n}+\sum_{k=1}^{n} \varphi_{m-k} \cdot\left\{\frac{\Gamma(\alpha)}{\Gamma(\alpha-k) \Gamma(k+1)}\left(z^{n}\right)_{k}\right. \\
& \left.+\frac{\Gamma(\alpha) n}{\Gamma(\alpha+1-k) \Gamma(k)}\left(z^{n-1}\right)_{k-1}\right\}=f \quad(z \neq 0)
\end{aligned}
$$

has a particular soution of the form

$$
\varphi=\left(f_{-\alpha} \cdot z^{-n}\right)_{\alpha-m}
$$

where $\varphi=\varphi(z), m \in \mathbb{Z}, n \in \mathbb{Z}^{+}, \alpha(\in \mathbb{C})$ is a given constant, $z \in \mathbb{C}$ and $f=f(z) \neq 0$ is known.

Proof. Setting

$$
\varphi=w_{\alpha} \quad\left(\varphi_{0}=\varphi\right)
$$

hence

$$
\varphi_{m}=w_{\alpha+m},
$$


we have then

$$
\begin{gathered}
w_{\alpha+m} \cdot z^{n}+\sum_{k=1}^{n} w_{\alpha+m-k}\left\{\frac{\Gamma(\alpha)}{\Gamma(\alpha-k) \Gamma(k+1)}\left(z^{n}\right) k\right. \\
\left.+\frac{\Gamma(\alpha) n}{\Gamma(\alpha+1-k) \Gamma(k)}\left(z^{n-1}\right)_{k-1}\right\}=f
\end{gathered}
$$

from (3.1). By the use of relationships (2.5) and (2.6), (3.4) gives

$$
\left(w_{m+1} \cdot z^{n}\right)_{\alpha-1}+n\left(w_{m} \cdot z^{n-1}\right)_{\alpha-1}=f
$$

that is,

$$
w_{m+1} \cdot z^{n}+n w_{m} \cdot z^{n-1}=f_{1-\alpha} .
$$

This is equivalent to

$$
\left(w_{m} \cdot z^{n}\right)_{1}=f_{1-\alpha}
$$

Thus we obtain

$$
w=\left(f_{-\alpha} \cdot z^{-n}\right)_{-m}
$$

or

$$
\varphi=w_{\alpha}=\left(f_{-\alpha} \cdot z^{-n}\right)_{\alpha-m}
$$

as a particular solution to the equation (3.1).

Conversely, we have

$$
\varphi_{m}=w_{\alpha+m}=\left(f_{-\alpha} \cdot z^{-n}\right)_{\alpha}
$$

then substituting this into the left hand side of (3.1), we obtain

L. H. S. of $(3.1)=\left(w_{m+1} \cdot z^{n}\right)_{\alpha-1}+n\left(w_{m} \cdot z^{n-1}\right)_{\alpha-1}$

$$
\begin{aligned}
& =\left(\left(f_{-\alpha} \cdot z^{-n}\right)_{1} \cdot z^{n}+n \cdot f_{-\alpha} \cdot z^{-n} \cdot z^{n-1}\right)_{\alpha-1} \\
& =\left(f_{1-\alpha}\right)_{\alpha-1} \\
& =f .
\end{aligned}
$$

Note 1. Equation (3.1) is a differential equation for $m \geq n$, a differintegral equation for $n>m>0$ and an integral equation for $m \leq 0$, respectively. 
Note 2. Letting $m=n$ in theorem 4 , we have theorem 1 in $\S 2$. That is,

$$
L[\varphi(z), n, n, \alpha]=L[\varphi(z), n, \alpha]=f \text { for } m=n .
$$

Theorem 5. Homogeous differintegral equation of Fuchs type

$$
L[\varphi(z), m, n, \alpha]=0 \quad(z \neq 0)
$$

has a solution

$$
\varphi=K\left(z^{-n}\right)_{\alpha-m}=K e^{-i \pi(\alpha-m)} \frac{\Gamma(\alpha-m+n)}{\Gamma(n)} z^{m-n-\alpha}
$$

where $m \in \mathbb{Z}, n \in \mathbb{Z}^{+},(\alpha-m+n) \notin \mathbb{Z}^{-} \cup\{0\}, z \in \mathbb{C}, \varphi=\varphi(z)$ and $K(\neq 0)$ is an arbitrary constant.

Proof. In the same way with the proof of previous theorem, with the aid of (3.3), (2.5) and (2.6), we obtain

$$
w_{m+1} \cdot z^{n}+n w_{m} \cdot z^{n-1}=0
$$

that is,

$$
\frac{w_{m+1}}{w_{m}}=-n z^{-1}
$$

from (3.5). Integrating both sides in (3.7), we have

$$
w_{m}=K z^{-n}, \quad(K \neq 0)
$$

hence

$$
\varphi=w_{\alpha}=K\left(z^{-n}\right)_{\alpha-m}=K e^{-i \pi(\alpha-m)} \frac{\Gamma(\alpha-m+n)}{\Gamma(n)} z^{m-n-\alpha},
$$

where $(\alpha-m+n) \notin \mathbb{Z}^{-} \cup\{0\}$.

Conversely, substiuting (3.8) into the left hand side of (3.5), we get

$$
\begin{aligned}
\text { L. II. S. of }(3.5) & =\left(w_{m+1} \cdot z^{n}+n w_{m} \cdot z^{n-1}\right)_{\alpha-1} r \\
& =\left(-K n z^{-1}+n K z^{-1}\right)_{\alpha-1} \\
& =(0)_{\alpha-1} \\
& =0 .
\end{aligned}
$$


Note. Letting $m=n$ in theorem 5 , we have theorem 2 in $\S 2$.

Theorem 6. If $f_{\alpha}(\neq 0)$ exists, then the differintegrated function

$$
\varphi=\left(f_{-\alpha} \cdot z^{-n}\right)_{\alpha-m}+K\left(z^{-n}\right)_{\alpha-m}
$$

satisfies the differintegral equation of Fuchs type (3.1), where $m \in \mathbb{Z}, n \in \mathbb{Z}^{+}$, $(\alpha-m+n) \notin \mathbb{Z}^{-} \cup\{0\}, z \in \mathbf{C}, \varphi=\varphi(z), f=f(z)$ is known, and $K(\neq 0)$ is an arbitrary constant.

Proof. It is clear by the theorems 1 and 2 .

Note. Letting $m=n$ in theorem 6 , we have theorem 3 in $\S 2$.

\section{References}

[1] K. Nishimoto, "Fractional derivative and integral", Part I, J. Coll. Engng. Nihon Univ. B-17, p. 11-19, (1976).

[2] - "Fractional differintegration of products", J. Coll. Engng. Nihon Univ. B-20, p. $1-7,(1979)$.

[3] - , "Table of fractional differintegration of elementary function:". J. Coll. Engng. Nihon Univ. B-25, p. 41-46, (1984).

[4] - - "Applications of fractional calculus to the solutions of linear second order differential equations of Fuch type", Research Notes in Math., 138, p. 140-153, (1985).

[5] - - "An application of fractional calculus to the non-homogeneous Gauss' equations", J. Coll. Engng. Nihon Univ., B-28, p. 1-8, (1987).

[6] K. Nishimoto and S. Owa: "Generalization of Nishimoto's Differential Equation", J. Coll. Engng. Nihon Univ., B-28, p. 15-19, (1987).

[7] S. Owa, K. Nishimoto, O. Altintas, S. K. Lee \& C. N. Eun; "Generalization of certain differential equation", Bull. Cal. Math. Soc. 80, p. 367-371, (1988).

$\left[8^{\circ}\right]$ K. Nishimoto; "Fractional Calculus", Vol. I, Descartes Press, Koriyama. Japan, 1984.

$\left[8^{b}\right]-$ - "Fractional Calculus", Vol. II, Descartes, 1987.

$\left[8^{c}\right]$ - - "Fractional Calculus", Vol. III, Descartes, 1989.

[9] K. B. Oldham and J. Spanier; "The Fractional Calculus", Academic Press (1974).

[10] B. Ross (Editor); "Lecture Notes in Mathematics". Vol. 457, Fractional Calculus and Its Applications. Springer (1975).

[11] A. C. McBride and G. F. Roach (Editors); "Research Notes in Mathematics". Vol. 138, Fractional Calculus, Pitman (1985).

[12] S. G. Samko, A. A. Kilbas and O. I. Marichev; "Fractional Integrals and Derivatives and Some of Their Applications", Nauka, USSR (1978).

[13] K. Nishimoto (Editor); "Fractional Calculus and Its Applications" International (Tokyo) Conference Proc. Coll. of Engng. Nihon Univ.. (1990). 
[14] Shih- Tong Tu, S-J Jaw and S-D Lin; "An application of fractional calculus to Chebyshev's equations". Chung Yuan J. Vol. XIX, p. 1-4, (1990).

Department of Mathematics, Chung Yuan Christian University, Chung Li, Taiwan, R.O.C.

Professor, College of Engineering, Nihon University, Koriyama, Japan. 